Pacific Journal of Mathematics

AN INVARIANCE PRINCIPLE FOR ASSOCIATED RANDOM 


\section{AN INVARIANCE PRINCIPLE FOR ASSOCIATED RANDOM FIELDS}

\section{Robert M. Burton, Jr. And TAe-Sung Kim}

Applying known tightness criteria to Poisson cluster random measures, it is shown that if the total member size has a finite $2+\delta$ moment, then the random measure satisfies an invariance principle.

I. Introduction. Let $\left\{X_{\underline{k}} \mid \underline{k} \in \mathbf{Z}^{d}\right\}$ be a random field that is centered, stationary, associated and has a summable covariance function. C. Newman [10] showed that, when viewed as an element in $d$-dimensional Skorohod space, the renormalizations of $\left\{X_{\underline{k}} \mid \underline{k} \in \mathbf{Z}^{d}\right\}$ converge to a Wiener measure in the sense of finite dimensional distributions. Newman and Wright [11] showed that this may be improved to an invariance principle if $d=1$ or 2 . Analogous results hold in the case of random measures. A tightness criterion of Bickel and Wichera [1] is applicable in the case of general $d$. This criterion is applied to Poisson center cluster random measures. It is shown that if the total member size has a finite $2+\delta$ moment then the random measure satisfies an invariance principle.

II. Random fields and random measures. A random field is a collection of nondegenerate random variables indexed by $\mathbf{Z}^{d}$ and is denoted $\left\{X_{k} \mid \underline{k} \in \mathbf{Z}^{d}\right\}$. All random fields in this section are assumed centered and stationary, i.e. $E\left[X_{\underline{k}}\right]=0$ and the distribution is invariant with respect to translations of the indices by the group $\mathbf{Z}^{d}$. A random field is associated if whenever $A \subseteq \mathbf{Z}^{d}$ is a finite subset and $f, g: \mathbf{R}^{A} \rightarrow \mathbf{R}$ are coordinatewise increasing then $\operatorname{Cov}\left[f\left(X_{k}: \underline{k} \in A\right), g\left(X_{k}: \underline{k} \in A\right)\right]$ is nonnegative whenever the covariance is defined. Association is a strong positive dependence property implying, in particular, nonnegative correlations of the random variables $X_{\underline{k}}$ (if they exist). For details concerning association see Esary, Proschan and Walkup [4].

A random field may be interpolated and rescaled to form a random element of $d$-dimensional Skorohod space $\mathfrak{D}\left([0,1]^{d}\right)$ by setting

$$
W_{n}(\underline{t})=n^{-d / 2} \sum_{j_{1}=1}^{\left[n t_{1}\right]} \cdots \sum_{j_{d}=1}^{\left[n t_{d}\right]} X_{\underline{j}}
$$


where $\underline{t}=\left(t_{1}, \ldots, t_{d}\right) \in[0,1]^{d}$ and [.] is the greatest integer function. $\left\{X_{\underline{k}}\right\}$ is said to satisfy an invariance principle or functional central limit theorem if $W_{n}(\underline{t})$ converges weakly in $\mathfrak{D}\left([0,1]^{d}\right)$ to a $d$-dimensional Wiener process with some finite diffusion constant $\sigma^{2}$. This is equivalent to convergence of the finite dimensional distributions together with tightness. The following condition arises naturally when investigating invariance principles.

Definition 2.1. $\left\{X_{k}\right\}$ has finite susceptibility equal to $\sigma^{2}<\infty$ if $\sum_{\underline{k} \in \mathbf{Z}^{d}} \operatorname{Cov}\left[X_{\underline{0}}, X_{\underline{k}}\right]=\sigma^{\frac{2}{2}}$.

REMARK. For $\underline{k} \in \mathbf{Z}^{d}$ with positive components let $S_{\underline{k}}=\sum_{\underline{0}<\underline{j} \leq \underline{k}} X_{\underline{j}}$. Assume that the covariances of the $X_{\underline{k}}$ are nonnegative. Then $\left\{\bar{X}_{\underline{k}}\right\}$ has finite susceptibility if and only if the following expectation is bounded in $\underline{k}$.

$$
E\left[\left.\left.\left|S_{\underline{k}} /\right| \underline{k}\right|^{1 / 2}\right|^{2}\right] \leq C
$$

where $|\underline{k}|=k_{1} \cdots k_{d}$.

The following theorem is due to Newman [10] and Newman and Wright [11].

THEOREM 2.2. Let $\left\{X_{\underline{k}}\right\}$ be an associated random field and have finite susceptibility equal to $\sigma^{2}$ then

(1) (Newman) the finite dimensional distributions of $W_{n}(\underline{t})$ converge to those of the Wiener process with diffusion $\sigma^{2}$;

(2) (Newman and Wright) further if $d=1$ or $d=2$ then $\left\{X_{\underline{k}}\right\}$ satisfies an invariance principle.

Whether an invariance principle holds for $d>2$ is still open. A result of Bickel and Wichura [1] allows us to conclude tightness if we strengthen the hypothesis of finite susceptibility.

DEFINITION 2.3. $\left\{X_{k}\right\}$ has finite $\delta$-susceptibility if there is a constant $C$ so that for all $\underline{k}$ with positive components

$$
E\left[\left.\left.\left|S_{\underline{k}} /\right| \underline{k}\right|^{1 / 2}\right|^{2+\delta}\right] \leq C .
$$

The above results may be combined to get the following.

THEOREM 2.4. Let $\left\{X_{\underline{k}}\right\}$ be a centered, stationary, finite variance, associated random field that satisfies $\delta$-susceptibility for some $\delta>0$. Then $\left\{X_{\underline{k}}\right\}$ satisfies the invariance principle. 
REMARK. The above theorem applies to models in mathematical physics. Random variables $X, Y, Z, W$ are said to satisfy the Lebowitz inequality if

$$
E[X Y Z W] \leq E[X Y] E[Z W]+E[X Z] E[Y W]+E[X W] E[Y Z] .
$$

A random field $\left\{X_{\underline{k}}\right\}$ satisfies the Lebowitz inequalities if any four coordinate random variables satisfy the above inequality. If $\left\{X_{\underline{k}}\right\}$ is also stationary and has finite susceptibility then it has finite $\delta$ susceptibility with $\delta=2$. This computation appears in Wood [18]. Ferromagnetic Ising models often satisfy the Lebowitz inequality and a fortiori satisfy the invariance principle. We have not been able to find this fact in the literature.

A corresponding theory exists for random measures. We let $M$ be the set of all nonnegative Borel measures on $\mathbf{R}^{d}$ that are finite on compact sets [i.e. Radon measures]. Let $N \subseteq M$ be the set of counting measures, i.e. $B$ Borel and $\mu \in N$ implies $\mu(B) \in\{0,1,2, \ldots, \infty\}$. There is a one-to-one correspondence between $\mu \in N$ and unordered sequences $\left\{x_{i}\right\}$ of points in $\mathbf{R}^{d}$ with no limit points because each such $\mu$ must be a sum of Dirac point masses. $M$ is a Polish space with the vague topology and $N$ is closed in $M$. Let the Borel $\sigma$-fields of $M$ and $N$ be $\mathscr{M}$ and $\mathscr{N}$ respectively.

Definition 2.5. A random measure $X$ is a measurable map from a fixed probability space $(\Omega, \mathscr{F}, P)$ to $(M, \mathscr{M}) . X$ is called a point random field if $P[X \in N]=1$.

If $X$ is a random measure and $B$ is a Borel subset of $\mathbf{R}^{d}$ then $X(B)$ denotes the mass the random measure gives to $B$. All random measures will be assumed to be stationary, i.e. with a translation invariant distribution. The most well known random measure is the Poisson point random field with parameter $\rho$. $X$ has this distribution if whenever $B_{1}, \ldots, B_{n}$ are disjoint bounded Borel sets then $X\left(B_{1}\right), \ldots, X\left(B_{n}\right)$ are independent Poisson random variables with respective parameters $\rho\left|B_{1}\right|, \ldots, \rho\left|B_{n}\right|$ where $|\cdot|$ denotes Lebesgue measure. $M$ has a partial ordering defined by $\mu \leq \nu$ if for each bounded Borel set $B$, $\mu(B) \leq \nu(B)$. See Kallenberg [6] for a more complete discussion of random measures.

DEFINITION 2.6. A random measure $X$ is associated if whenever $F, G: M \rightarrow \mathbf{R}$ is measurable and increasing with respect to the partial ordering on $M$ then $\operatorname{Cov}[F(X), G(X)]$ is nonnegative whenever the 
covariance is defined. It follows from work of the first author and Waymire [2,3] that $X$ is associated if and only if the family of random variables $\{X(B) \mid B$ bounded Borel $\}$ is associated.

DEFINITION 2.7. If $X$ is a random measure we define the $\lambda$-renormalization of $X$ to be the signed random measure $X_{\lambda}$ where $X_{\lambda}(B)=$ $\lambda^{-d / 2}[X(\lambda B)-E[X(\lambda B)]]$. We consider $X_{\lambda}$ as a random element of $\mathfrak{D}\left([0,1]^{d}\right)$ by setting $X_{\lambda}(\underline{t})=X_{\lambda}([\underline{0}, \underline{t}])$ where $[\underline{0}, \underline{t}]$ is the rectangle $\left[0, t_{1}\right] \times \cdots \times\left[0, t_{d}\right]$.

Definition 2.8. $X$ satisfies the invariance principle with parameter $\sigma^{2}$ if as $\lambda \rightarrow \infty, X_{\lambda}$ converges weakly to the $d$-dimensional Wiener measure on $\mathfrak{D}\left([0,1]^{d}\right)$ with diffusion constant $\sigma^{2}$.

Definition 2.9. Let $I$ be the unit cube in $\mathbf{R}^{d}$ and $X$ be associated. $X$ has finite susceptibility $\sigma^{2}$ if

$$
\sum_{\underline{k} \in \mathbf{Z}^{d}} \operatorname{Cov}[X(I), X(I+\underline{k})]=\sigma^{2} .
$$

$X$ has finite $\delta$-susceptibility if there is a constant $K<\infty$ depending only on $\delta$ and $X$ so that for all rectangular boxes $B \supseteq I$ we have

$$
E\left[|X(B)-E[X(B)]|^{2+\delta}\right] \leq K|B|^{1+\delta / 2} .
$$

It would be a more pleasing definition to require $(*)$ in Definition 2.9 to hold for all rectangular boxes $B$ but this is asking too much. For example such a condition is untrue in the case where $X$ is a Poisson point random field for any $\delta>0$. A simple argument using Chebyshev's inequality allows us to extend the invariance principle for associated random fields to random measures.

THEOREM 2.10. Let $X$ be a stationary associated random measure with finite $\delta$-susceptibility for some $\delta>0$. Then $X$ satisfies the invariance principle.

III. Cluster random measures. In this section we apply Theorem 2.10 to Poisson center cluster random measures. These have been used as models of infinite divisibility and self-similarity $[14,15]$ as well as models of natural phenomena such as storm systems and galaxies $[12,16,17]$. These are constructed as follows. Let $U$ be a stationary Poisson point random field with parameter $\rho$. Let $V=\left\{V_{x} \mid \underline{x} \in \mathbf{R}^{d}\right\}$ 
be a collection of iid random measures with $E\left[V_{\underline{x}}\left(\mathbf{R}^{d}\right)\right]=\xi<\infty$. Then we say that $X$ is a cluster process with centers $U$ and members $V$ if

$$
X(B)=\sum_{\underline{x}: U(\underline{x})>0} V_{\underline{x}}(B-\underline{x})
$$

for each bounded Borel set $B$. We denote $X$ by $[U, V]$. It is natural to hope that moment conditions on $V$ will imply moment conditions on $X$ regardless of the "shape" of $V$ in $\mathbf{R}^{d}$. This is made precise in the following theorem.

THEOREM 3.1. Let $X=[U, V]$ as above. Let $B$ be a rectangular box in $\mathbf{R}^{d}$ and $0 \leq \delta \leq 2$; then there is a constant $K$ depending only on $\delta$ and $|B|$ so that

(1) $E\left[|X(B)|^{2+\delta}\right] \leq K E\left[\left(V_{\underline{x}}\left(\mathbf{R}^{d}\right)\right)^{2+\delta}\right]$.

(2) If $E\left[\left(V_{\underline{x}}\left(\mathbf{R}^{d}\right)\right)^{2+\delta}\right]<\infty$ then $X$ has finite $\delta$-susceptibility.

The first part of the next theorem appears in joint work of the first author and Waymire [2] and the second part is immediate from the first part of Theorem 3.1.

THEOREM 3.2. Let $X=[U, V]$ as above.

(1) $X$ is associated.

(2) If $E\left[\left(V_{\underline{x}}\left(\mathbf{R}^{d}\right)\right)^{2+\delta}\right]<\infty$ then $X$ satisfies the invariance principle.

We note that the second part of Theorem 3.2 improves a theorem of Ivanoff [5] where it was assumed that $\delta=4$ and that the $V$ was a point random field with cumulant density functions.

IV. Proof of Theorem 3.1. First two lemmas.

LEMMA 4.1. (1) If $Y_{1}, Y_{2}, \ldots$ are centered iid random variables and $E\left|Y_{i}\right|^{p}<\infty$ for some $p \geq 1$ then there is a constant $C$ depending only on $p$ so that

$$
E\left|\sum_{i=1}^{n} Y_{i}\right|^{p} \leq C E\left|\sum_{i=1}^{n} Y_{i}^{2}\right|^{p / 2}
$$

(2) If $Y_{1}, Y_{2}, \ldots$ are iid random variables and $E\left|Y_{i}\right|^{2+\delta}<\infty$ for some $0 \leq \delta \leq 2$ then there is a constant $C^{\prime}$ depending only on $\delta$ so that

$$
E\left|\sum_{i=1}^{n} Y_{i}\right|^{2+\delta} \leq C^{\prime}\left(n E\left|Y_{1}\right|^{2+\delta}+\left|n E Y_{1}^{2}\right|^{1+\delta / 2}+\left|n E Y_{1}\right|^{2+\delta}\right)
$$


Proof. (1) is a standard square inequality attributed to Marcinkiewicz and Zygmund [9] (see also [13], p. 59). (2) follows from two applications of (1). Let $\mu=E\left[Y_{1}\right]$ and $\sigma^{2}=\operatorname{Var}\left[Y_{1}\right]$ and $\stackrel{\sim}{\leq}$ denote "bounded by a constant multiple of". Then

$$
\begin{aligned}
& E\left|\sum_{i=1}^{n} Y_{i}\right|^{2+\delta} \stackrel{\sim}{\leq} E\left|\sum_{i=1}^{n}\left(Y_{i}-\mu\right)\right|^{2+\delta}+|n \mu|^{2+\delta} \\
& \underset{\leq}{\leq} E\left|\sum_{i=1}^{n}\left(Y_{i}-\mu\right)^{2}\right|^{1+\delta / 2}+|n \mu|^{2+\delta}
\end{aligned}
$$

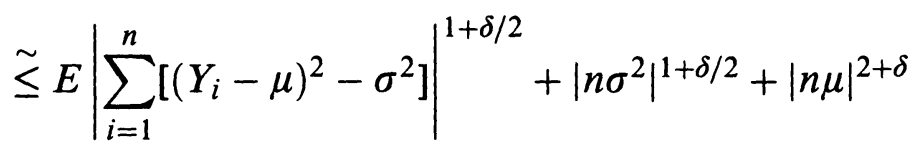

$$
\begin{aligned}
& \stackrel{\sim}{\leq} E\left|\sum_{i=1}^{n}\left[\left(Y_{i}-\mu\right)^{2}-\sigma^{2}\right]^{2}\right|^{1 / 2+\delta / 4} \\
& +\left|n \sigma^{2}\right|^{1+\delta / 2}+|n \mu|^{2+\delta} \\
& \tilde{\leq} E \sum_{i=1}^{n}\left|\left[\left(Y_{i}-\mu\right)^{2}-\sigma^{2}\right]^{2}\right|^{1 / 2+\delta / 4} \\
& +\left|n \sigma^{2}\right|^{1+\delta / 2}+|n \mu|^{2+\delta} \\
& =n E\left|\left[\left(Y_{1}-\mu\right)^{2}-\sigma^{2}\right]\right|^{1+\delta / 2}+\left|n \sigma^{2}\right|^{1+\delta / 2}+|n \mu|^{2+\delta} \\
& \widetilde{\leq} n E\left|Y_{1}-\mu\right|^{2+\delta}+n\left|\sigma^{2}\right|^{1+\delta / 2}+\left|n \sigma^{2}\right|^{1+\delta / 2}+|n \mu|^{2+\delta} \\
& \stackrel{\sim}{\leq} E\left|Y_{1}\right|^{2+\delta}+n\left(E\left[Y_{1}^{2}\right]\right)^{1+\delta / 2} \\
& +\left(n E\left[Y_{1}^{2}\right]\right)^{1+\delta / 2}+\left|n E Y_{1}\right|^{2+\delta} \\
& \stackrel{\sim}{\leq} E\left|Y_{1}\right|^{2+\delta} \\
& +\left|n E Y_{1}^{2}\right|^{1+\delta / 2}+\left|n E Y_{1}\right|^{2+\delta} \text {. }
\end{aligned}
$$

LEMMA 4.2. Let $V=\left\{V_{\underline{x}} \mid \underline{x} \in \mathbf{R}^{d}\right\}$ be iid random measures as in the definition of a cluster random measure. Let $B \subseteq \mathbf{R}^{d}$ be a rectangle in the nonnegative orthant with one corner at the origin and the opposite corner at $\underline{z}$ and let $B^{(n)}=\bigcup_{\underline{k}: n \leq k_{1}<n} B+\underline{z}(\underline{k})$, where $\underline{z}(\underline{k})$ is the vector with ith coordinate $k_{i} z_{i}$. Let $\underline{x}$ be chosen uniformly in $B^{(n)}$. Then for $\alpha \geq 1$

$$
E\left|V_{\underline{x}}(B-\underline{x})\right|^{\alpha} \leq 2^{-d} n^{-d} e\left[V_{\underline{x}}\left(\mathbf{R}^{d}\right)^{\alpha}\right]
$$


Proof.

$$
\begin{aligned}
E\left|V_{\underline{x}}(B-\underline{x})\right|^{\alpha}=E\left[\frac{1}{\left|B^{(n)}\right|} \int_{B^{(n)}} V_{\underline{x}}(B-\underline{x})^{\alpha} d \underline{x}\right] \\
=E\left[\frac{1}{2^{d} n^{d}|B|} \sum_{\underline{k}: n \leq k_{i}<n} \int_{B+\underline{z}(\underline{k})} V_{\underline{x}}(B-\underline{x})^{\alpha} d \underline{x}\right] \\
=E\left[2^{-d} n^{-d}|B|^{-1} \sum_{\underline{k}: n \leq k_{i}<n} \int_{B} V_{\underline{x}}(B-\underline{x}-\underline{z}(\underline{k}))^{\alpha} d \underline{x}\right] \\
\leq E\left[2^{-d} n^{-d}|B|^{-1} \int_{B}\left(\sum_{\underline{k}: n \leq k_{i}<n} V_{\underline{x}}(B-\underline{x}-\underline{z}(\underline{k}))^{\alpha} d \underline{x}\right]\right. \\
\leq 2^{-d} n^{-d}|B|^{-1} E \int_{B} V_{\underline{x}}\left(\mathbf{R}^{d}\right)^{\alpha} d \underline{x}=2^{-d} n^{-d} E\left[V_{\underline{x}}\left(\mathbf{R}^{d}\right)^{\alpha}\right] .
\end{aligned}
$$

Proof of Theorem 3.1. Let $X=[U, V]$ as in the statement of the theorem and suppose that $\delta \leq 2$ and that $E\left[V\left(\mathbf{R}^{d}\right)^{2+\delta}\right]<\infty$. Let $R_{n}$ be equal to the number of occurrences of $U$ in $B^{(n)}$ so that $R_{n}$ is a Poisson random variable with parameter $\rho 2^{d} n^{d}|B|$. Also recall that conditioned on $R_{n}$ the occurrences of $U$ inside $B^{(n)}$ are independent and uniformly distributed on $B^{(n)}$.

$$
\begin{aligned}
E|X(B)|^{2+\delta}= & \lim _{n \rightarrow \infty}\left[\left(\sum_{\underline{x}_{i} \in B^{(n)}, U\left(\underline{x}_{i}\right)>0} V_{\underline{x}_{i}}\left(B-\underline{x}_{i}\right)\right)^{2+\delta}\right] \\
= & \lim _{n \rightarrow \infty} E\left[E\left[\left(\sum V_{\underline{x}_{i}}\left(B-\underline{x}_{i}\right)\right)^{2+\delta} \mid R_{n}\right]\right] \\
& \tilde{\leq} \lim _{n \rightarrow \infty} E\left[R_{n} E\left|V_{\underline{x}}(B-\underline{x})\right|^{2+\delta}+\left|R_{n} E V_{\underline{x}}(B-\underline{x})^{2}\right|^{1+\delta / 2}\right. \\
& \left.\quad+\left|R_{n} E V_{\underline{x}}(B-\underline{x})\right|^{2+\delta}\right] \\
& \simeq \lim _{n \rightarrow \infty} \rho 2^{d} n^{d}|B| 2^{-d} n^{-d} E\left[V\left(\mathbf{R}^{d}\right)^{2+\delta}\right] \\
& +\left(\rho 2^{d} n^{d}|B|\right)^{1+\delta / 2}\left(2^{-d} n^{-d} E\left[V\left(\mathbf{R}^{d}\right)^{2}\right]\right)^{1+\delta / 2} \\
& +\left(\rho|B| E\left[V\left(\mathbf{R}^{d}\right)\right]\right)^{2+\delta} \stackrel{\sim}{\leq} E\left[V\left(\mathbf{R}^{d}\right)^{2+\delta}\right] .
\end{aligned}
$$

Note that the factor $\left(\rho 2^{d} n^{d}|B|\right)^{1+\delta / 2}$ in the second term follows from the fact that if $R$ is Poisson with parameter $\alpha$ then $E\left[R^{1+\delta / 2}\right] \leq$ const. $\alpha^{1+\delta / 2}$ for nonnegative $\delta$. This proves part (1). To see part 
(2) which is finite $\delta$-susceptibility we have a final computation.

$$
\begin{aligned}
& E\left[|X(B)-E[X(B)]|^{2+\delta}\right] \\
& =\lim _{n \rightarrow \infty} E\left[\left|\sum_{\underline{x}_{i} \in B^{(n)}, U\left(\underline{x}_{i}\right)>0} V_{\underline{x}_{i}}\left(B-\underline{x}_{i}\right)-\sum V_{\underline{x}_{i}}\left(B-\underline{x}_{i}\right)\right|^{2+\delta}\right] \\
& \leq \lim _{n \rightarrow \infty} E\left[E\left[\left|\sum V_{\underline{x}_{i}}\left(B-\underline{x}_{i}\right)-E\left[\underline{V}_{\underline{x}_{i}}\left(B-\underline{x}_{i}\right)\right]\right|^{2+\delta} \mid R_{n}\right]\right] \\
& \quad+\lim _{n \rightarrow \infty} E\left[\left|R_{n} E\left[{\underline{V_{x}}}_{i}\left(B-\underline{x}_{i}\right)\right]-E R_{n} E\left[V_{\underline{x}_{i}}\left(B-\underline{x}_{i}\right)\right]\right|^{2+\delta}\right] .
\end{aligned}
$$

The second term is the limit of $E\left(\left|R_{n}-E R_{n}\right|^{2+\delta}\right)\left(E\left[V_{x_{i}}\left(B-\underline{x}_{i}\right)\right]\right)^{2+\delta}$ which, by part (1) and some Poisson distribution calculations, is $\stackrel{\sim}{\leq}\left(\rho 2^{d} n^{d}|B|\right)^{1+\delta / 2}\left(2^{-d} n^{-d} E\left[V\left(\mathbf{R}^{d}\right)\right]\right)^{2+\delta}$ which goes to zero. This leaves us with the first term which is by Lemma 4.1(2)

$$
\begin{aligned}
& \lim _{n \rightarrow \infty} E\left[R_{n} E\left|V_{\underline{x}_{i}}\left(B-\underline{x}_{i}\right)-E\left[V_{\underline{x}_{i}}\left(B-\underline{x}_{i}\right)\right]\right|^{2+\delta}\right. \\
& \left.+R_{n}^{1+\delta / 2}\left|E\left[V_{\underline{x}_{i}}\left(B-\underline{x}_{i}\right)^{2}\right]\right|^{1+\delta / 2}\right] \\
& \tilde{\leq} \lim _{n \rightarrow \infty} E\left[R_{n} E\left|V_{\underline{x}_{i}}\left(B-\underline{x}_{i}\right)\right|^{2+\delta}+R_{n}^{1+\delta / 2}\left|E\left[V_{\underline{x}_{i}}\left(B-\underline{x}_{i}\right)^{2}\right]\right|^{1+\delta / 2}\right] \\
& \tilde{\leq} \lim _{n \rightarrow \infty} \rho 2^{d} n^{d}|B| 2^{-d} n^{-d} E\left[V\left(\mathbf{R}^{d}\right)^{2+\delta}\right] \\
& +\rho^{1+\delta / 2}|B|^{1+\delta / 2}\left(E\left[V\left(\mathbf{R}^{d}\right)^{2}\right]\right)^{1+\delta / 2} \\
& \stackrel{\sim}{\leq}|B|^{1+\delta / 2} E\left[V\left(\mathbf{R}^{d}\right)^{2+\delta}\right] \text {. }
\end{aligned}
$$

Acknowledgments. The authors wish to thank the referee for a very thorough review of this paper. Part of this work appears in the Oregon State University doctoral dissertation of the second author, who gratefully acknowledges numerous helpful discussions with Ed Waymire.

\section{REFERENCES}

[1] P. J. Bickel and M. J. Wichura, Convergence criteria for multiparameter stochastic processes and some applications, Ann. Math. Stat., 42 (1971), 1650-1670.

[2] R. M. Burton and E. Waymire, Scaling limits for associated random measures, Ann. Probab., 13 (1985), 1267-1278.

[3] R. M. Burton and E. Waymire, On the central limit problem for infinitely divisible random measures, In Dependence in Probability and Statistics, p. 383-395. ed. E. Eberlein and M. Taqqu, Birkhäuser (1986).

[4] J. Esary, F. Proschan and D. Walkup, Association of random variables with applications, Ann. Math. Stat., 38 (1967), 1466-1474.

[5] G. Ivanoff, Central limit theorems for point processes, Stoch. Proc. Appl., 12 (1982), 171-186. 
[6] O. Kallenberg, Random Measures, (1976) Academic Press, New York.

[7] J. Kerstan, K. Matthes and J. Mecke, Infinitely Divisible Point Processes, (1978) Wiley, New York.

[8] J. Lebowitz, Bounds on the correlations and analyticity properties of ferromagnetic Ising spin systems, Comm. Math. Phys., 28 (1972), 311-321.

[9] J. Marcinkiewicz and A. Zygmund, Sur les fonctions indépendantes, Fund Math., 29 (1937), 60-90.

[10] C. M. Newman, Normal fluctuations and the FKG inequalities, Comm. Math. Phys., 74 (1980), 119-128.

[11] C. M. Newman and A. L. Wright, An invariance principle for certain dependent sequences, Ann. Probab., 9 (1981), 671-765.

[12] J. Neyman and E. L. Scott, A statistical approach to problems of cosmology, J. Roy. Statist. Soc. ser. B, 20 (1958), 1-43.

[13] V. V. Petrov, Some of Independent Random Variables, (1975) Springer-Verlag, New York.

[14] D. Surgalis, On infinitely divisible self-similar random fields, Z. Wahrsch. Verw. Gebiete., 58 (1981), 453-477.

[15] M. S. Taqqu and R. L. Wolpert, Infinite variance self-similar processes subordinate to a Poisson measure, Z. Wahrsch. Verw. Gebiete., 62 (1983), 53-72.

[16] D. Vere-Jones, Stochastic models for earthquake occurrence, J. Roy. Statist. Soc. ser. B, 32 (1970), 1-62.

[17] E. Waymire, V. K. Gupta and I. Rodriguez, $A$ spectral theory of rainfall at the meso- $\beta$ scale, Water Res. J., 20 (1984), 1453-1465.

[18] T. E. Wood, A Berry-Esseen theorem for associated random variables, Ann. Probab., 11 (1983), 1042-1047.

Received July 31, 1986 and in revised form May 12, 1987. The first author was supported in part by NSF grant MCS-8005172. The second author was supported in part by a KRF grant in 1986 .

OREgon State University

CORVALLIS, OR 97331

AND

WON KWANG UNIVERSITY

IRI City, RePUbLiC OF Korea 



\section{PACIFIC JOURNAL OF MATHEMATICS \\ EDITORS}

V. S. VARADARAJAN

(Managing Editor)

University of California

Los Angeles, CA 90024

Herbert Clemens

University of Utah

Salt Lake City, UT 84112

R. FINN

Stanford University

Stanford, CA 94305
HERMANN FLASCHKA

University of Arizona

Tucson, AZ 85721

RAMESH A. GANGOLLI

University of Washington Seattle, WA 98195

VAUGHAN F. R. JONES

University of California

Berkeley, CA 94720
ROBION KIRBY

University of California

Berkeley, CA 94720

C. C. MOORE

University of California

Berkeley, CA 94720

HAROLD STARK

University of California, San Diego

La Jolla, CA 92093

\section{ASSOCIATE EDITORS}
R. ARENS
E. F. BECKENBACH
B. H. NEUMANN
F. WOLF
K. YOSHIDA
(1906-1982)

\section{SUPPORTING INSTITUTIONS}

UNIVERSITY OF ARIZONA

UNIVERSITY OF BRITISH COLUMBIA

CALIFORNIA INSTITUTE OF TECHNOLOGY

UNIVERSITY OF CALIFORNIA

MONTANA STATE UNIVERSITY

UNIVERSITY OF NEVADA, RENO

NEW MEXICO STATE UNIVERSITY

OREGON STATE UNIVERSITY
UNIVERSITY OF OREGON UNIVERSITY OF SOUTHERN CALIFORNIA

STANFORD UNIVERSITY

UNIVERSITY OF HAWAII

UNIVERSITY OF TOKYO

UNIVERSITY OF UTAH

WASHINGTON STATE UNIVERSITY

UNIVERSITY OF WASHINGTON 


\section{Pacific Journal of Mathematics \\ Vol. 132, No. $1 \quad$ January, 1988}

Scott W. Brown, Full analytic subspaces for contractions with rich spectrum ...1

Robert Main Burton, Jr. and Tae-Sung Kim, An invariance principle for

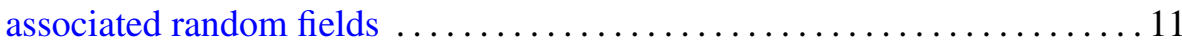

Ana M. Viola-Prioli and Jorge Viola-Prioli, Rings whose kernel functors

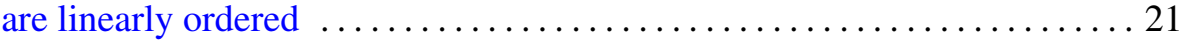

David E. Handelman, Representing polynomials by positive linear

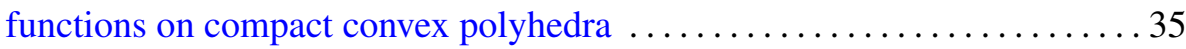

Patrick Keef, On the Tor functor and some classes of abelian groups . .....66

Dennis R. Malm, Simplicity of partial and Schmidt differential operator

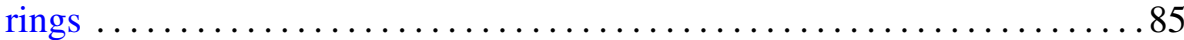

José M. Montesinos and Carmen Safont, On the Birman invariants of

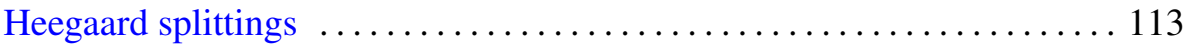

N. P. Mukherjee and Prabir Bhattacharya, The normal index of a finite group

Mario Petrich and Norman R. Reilly, Semigroups generated by certain operators on varieties of completely regular semigroups

Robert Tijdeman and Lian Xiang Wang, Sums of products of powers of given prime numbers

Joel Larry Weiner, First integrals for a direction field on a simply connected plane domain 